\title{
Quantum Interference Effects on Optical Amplification and the Index of Refraction in a Four-Level System
}

\author{
Hui-Fang Zhang*, Jin-Hui Wu, and Jin-Yue Gao \\ College of Physics, Jilin University, \\ Key Lab of Coherent Light, Atomic and Molecular Spectroscopy, \\ Educational Ministry of China, ChangChun, CHINA
}

(Received February 26, 2003)

\begin{abstract}
We construct a four-level system where a metastable state is included in an $\mathrm{Er}^{3+}$ Doped Yttrium aluminum garnet (YAG) crystal. Because of the action of the coherent field, the traditional light amplification with inversion can be exhibited with remarkable variation. As a result, we propose a method to achieve the gain equalization by atomic coherence. At the same time, we find that the high index of refraction accompanied by vanishing absorption can also be reached in this model. We also find that a higher index of refraction with zero absorption can be easily obtained when the coherent field is off resonance.
\end{abstract}

OCIS codes : $270.1670 ; 270.3430 ; 060.2320$.

\section{INTRODUCTION}

Recent studies on effects of atomic coherence and quantum interference in quantum optics has led to many interesting optical phenomena such as electromagnetically induced transparency (EIT) [1-4], laser without inversion (LWI) $[5,6]$, enhancement of refractive index [7-9] and so on. Up to now, many experimental and theoretical studies of quantum interference have been focused on atomic gas media [10-12]. For the understanding of quantum coherence and interference in crystals and for various potential device applications, EIT in solid media become more and more attractive [13-15].

In this paper, we investigate a four-level scheme in an $\mathrm{Er}^{3+}$-doped Yttrium aluminum garnet (YAG) crystal to discuss the effects of quantum interference on optical amplification and the index of refraction. Based on the mechanism of atomic coherence, we suggest a method to get probe gain equalization, which may be used in optical fiber communication. At the same time, we also find that the high index of refraction with zero absorption can be achieved in this system, the index-enhanced media has potential application in laser particle acceleration, optical microscopy, atomic tests of electroweak physics, and magnetometry etc. By modulating the incoherent pumping and the coherent field, we see that the position and value of gain peaks, the height and width of the equalized gain, and the maximum value of index of refraction with vanishing absorption all are changed correspondingly.

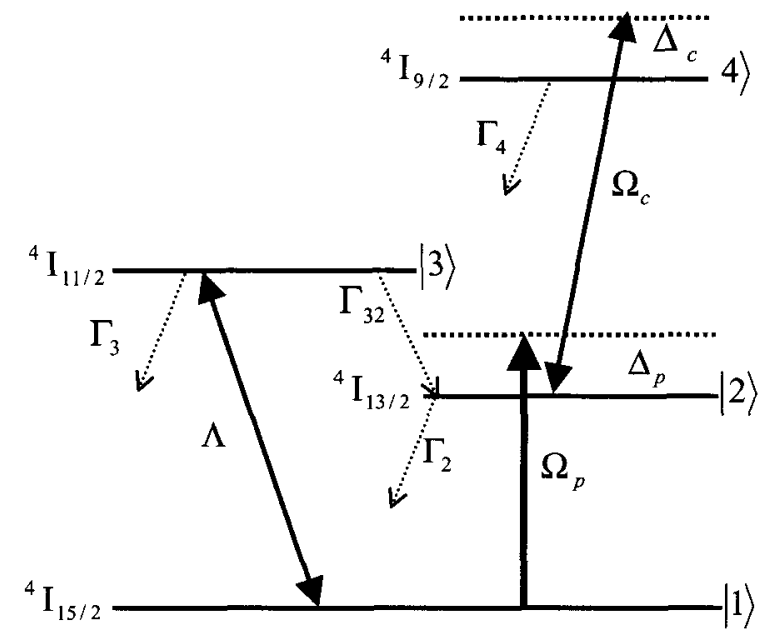

FIG. 1. Schematic diagram of a four-level system where levels $|1\rangle,|2\rangle,|3\rangle$ and $|4\rangle$ correspond to energy levels of $\mathrm{Er}^{3+}$ ions in ZBLAN glass ${ }^{4} \mathrm{I}_{15 / 2},{ }^{4} \mathrm{I}_{13 / 2},{ }^{4} \mathrm{I}_{11 / 2}$ and ${ }^{4} I_{9 / 2}$, respectively. Levels $|1\rangle,|2\rangle$, and $|4\rangle$ form a EIT subsystem, while levels $|1\rangle,|3\rangle$ and $|2\rangle$ form a light amplification subsystem. 


\section{THE MODEL AND EQUATIONS}

We consider a closed four-level system shown in Fig. 1. The energy-level scheme is relevant to the $\mathrm{Er}^{3+}$-doped Yttrium aluminum garnet (YAG) crystal, where levels $|1\rangle,|2\rangle,|3\rangle$ and $|4\rangle$ correspond to energy levels of $\mathrm{Er}^{3+}$ ions ${ }^{4} \mathrm{I}_{15 / 2},{ }^{4} \mathrm{I}_{13 / 2},{ }^{4} \mathrm{I}_{11 / 2},{ }^{4} \mathrm{I}_{9 / 2}$, respectively. In this system, via a fast decay from $|3\rangle$, level $|2\rangle$ is populated by an incoherent pump process $\Lambda$ interacting with the transition $|1\rangle \leftrightarrow|3\rangle$. A coherent driving field $\mathrm{E}_{c}$ with Rabi frequency $\Omega_{c}$ drives the $|2\rangle \leftrightarrow|4\rangle$ atomic transition, and a probe field $\mathrm{E}_{p}$ with Rabi frequency $\Omega_{p}$ interacts with the transition labeled $|1\rangle \leftrightarrow|2\rangle . \Gamma_{4}=\Gamma_{41}+\Gamma_{42}+\Gamma_{43}$ is the spontaneous decay rate of level $|4\rangle, \Gamma_{3}=\Gamma_{31}+\Gamma_{32}$ is the spontaneous decay rate of level $|3\rangle, \Gamma_{2}=\Gamma_{21}$ is the spontaneous decay rate of level $|2\rangle$. When the incoherent pump rate $\Lambda=0$, it is obvious that this system can be treated as a three-level ladder-type EIT system composed of levels $|1\rangle,|2\rangle$ and $|4\rangle$, while when the Rabi frequency of the coherent field $\Omega_{c}=0$, it is a traditional optical amplification system with population inversion.

In the framework of the semiclassical theory and using the standard density matrix formalism with the dipole approximation and the rotating wave approximation, the description equations of this system can be written as:

$$
\begin{aligned}
& \rho_{11}=\Lambda\left(\rho_{33}-\rho_{11}\right)+\Gamma_{21} \rho_{22}+\Gamma_{31} \rho_{33}+\Gamma_{41} \rho_{44}+i \Omega_{p}^{*} \rho_{21}-i \Omega_{p} \rho_{12} \\
& \rho_{22}=-\Gamma_{21} \rho_{22}+\Gamma_{32} \rho_{33}+\Gamma_{42} \rho_{44}+i \Omega_{p} \rho_{12}-i \Omega_{p}^{*} \rho_{21}+i \Omega_{c}^{*} \rho_{42}-i \Omega_{c} \rho_{24} \\
& \rho_{33}=-\Lambda\left(\rho_{33}-\rho_{11}\right)-\Gamma_{31} \rho_{33}-\Gamma_{32} \rho_{33}+\Gamma_{43} \rho_{44} \\
& \rho_{41}=-\left[\gamma_{41}-i\left(\Delta_{p}+\Delta_{c}\right)\right] \rho_{41}+i \Omega_{c} \rho_{21}-i \Omega_{p} \rho_{42} \\
& \rho_{42}=-\left(\gamma_{42}-i \Delta_{c}\right) \rho_{42}+i \Omega_{c}\left(\rho_{22}-\rho_{44}\right)-i \Omega_{p}^{*} \rho_{41} \\
& \rho_{43}=-\left[\gamma_{43}-i\left(\Delta_{p}+\Delta_{c}\right)\right] \rho_{43}+i \Omega_{c} \rho_{23} \\
& \rho_{32}=-\left(\gamma_{32}+i \Delta_{p}\right) \rho_{32}-i \Omega_{p}^{*} \rho_{31}-i \Omega_{c} \rho_{34} \\
& \rho_{31}=-\gamma_{31} \rho_{31}-i \Omega_{p} \rho_{32} \\
& \rho_{21}=-\left(\gamma_{21}-i \Delta_{p}\right) \rho_{21}+i \Omega_{p}\left(\rho_{11}-\rho_{22}\right)+i \Omega_{c}^{*} \rho_{41} \\
& \rho_{11}+\rho_{22}+\rho_{33}+\rho_{44}=1 \\
& \rho_{i j}=\rho_{j i}^{*}
\end{aligned}
$$

Where, $\Delta_{p}$ and $\Delta_{c}$ describe the detunings of the probe and the coherent field, respectively. $\Gamma_{i j}$ designates the population spontaneous damping from $|i\rangle$ to $|j\rangle, \gamma_{i j}$ are total coherence relaxation rates between $|i\rangle$ and $|j\rangle$, given by [16]:

$$
\begin{aligned}
\gamma_{41} & =\left(\Gamma_{41}+\Gamma_{42}+\Gamma_{43}+\Lambda+\gamma_{41}^{d p h}\right) / 2 \\
\gamma_{42} & =\left(\Gamma_{41}+\Gamma_{42}+\Gamma_{43}+\Gamma_{21}+\gamma_{42}^{d p h}\right) / 2 \\
\gamma_{43} & =\left(\Gamma_{41}+\Gamma_{42}+\Gamma_{43}+\Gamma_{31}+\Gamma_{32}+\Lambda+\gamma_{43}^{d p h}\right) / 2 \\
\gamma_{32} & =\left(\Gamma_{32}+\Gamma_{31}+\Gamma_{21}+\Lambda+\gamma_{32}^{d p h}\right) / 2 \\
\gamma_{31} & =\left(\Gamma_{32}+\Gamma_{31}+\Lambda+\Lambda+\gamma_{31}^{d p h}\right) / 2 \\
\gamma_{21} & =\left(\Gamma_{21}+\Lambda+\gamma_{21}^{d p h}\right) / 2
\end{aligned}
$$

where $\gamma_{i j}^{d p h}$ is the dephasing decay rate of the quantum coherence of the $|i\rangle \leftrightarrow|j\rangle$ transition. In contrast to many atomic schemes the $\gamma_{i j}^{d p h}$, determined by electron-electron, interface roughness, and phonon scattering processes, are the dominant contributions to the $\gamma_{i j}$ and the major obstacle to the observation of coherent effects such as EIT in solid material.

\section{ANALYTICAL DISCUSSIONS}

In the limit of a weak probe, under the steady-state condition, the solutions of Eq. (1) for $\rho_{21}$ to the first order of the probe field and to the zero order for other $\rho_{i j}$ are:

$$
\begin{aligned}
\rho_{22} & =\frac{\Lambda \Gamma_{32} /\left(\Lambda+\Gamma_{31}+\Gamma_{32}\right)}{\left[\Gamma_{21}+\left(\Gamma_{41}+\frac{\Gamma_{43}\left(\Lambda+\Gamma_{31}\right)}{\Lambda+\Gamma_{31}+\Gamma_{32}}\right) \frac{D_{2}}{D_{1}}\right]} \rho_{11} \\
\rho_{33} & =\left[\Gamma_{21}+\left(\Gamma_{41}+\Gamma_{43}\right) \frac{D_{2}}{D_{1}}\right] \frac{\rho_{22}}{\Gamma_{32}} \\
\rho_{44} & =\frac{D_{2}}{D_{1}} \rho_{22} \\
\rho_{42} & =\frac{i \Omega_{c}\left(\rho_{22}-\rho_{44}\right)}{\gamma_{42}-i \Delta_{c}}
\end{aligned}
$$

and 


$$
\rho_{12}=\rho_{21}^{*}=\frac{i \Omega_{p}\left(\rho_{22}-\rho_{11}\right)-\frac{i\left|\Omega_{c}\right|^{2} \Omega_{p}\left(\rho_{22}-\rho_{44}\right)}{\left[\gamma_{41}+i\left(\Delta_{p}+\Delta_{c}\right)\right]\left(\gamma_{42}+i \Delta_{c}\right)}}{\gamma_{21}+i \Delta_{p}+\frac{\left|\Omega_{c}\right|^{2}}{\gamma_{41}+i\left(\Delta_{p}+\Delta_{c}\right)}}
$$

$$
\begin{aligned}
& P=2 N \mu_{21} \rho_{12} \\
& \operatorname{Im} P=2 N \mu_{21} \operatorname{Im}\left(\rho_{12}\right) \\
& \operatorname{Re} P=2 N \mu_{21} \operatorname{Re}\left(\rho_{12}\right)
\end{aligned}
$$

where $D_{1}=\Gamma_{41}+\Gamma_{42}+\Gamma_{43}+\frac{2\left|\Omega_{c}\right|^{2} \gamma_{42}}{\gamma_{42}^{2}+\Delta_{c}^{2}}, D_{2}=\frac{2 \mid \Omega_{c}{ }^{2} \gamma_{42}}{\gamma_{42}^{2}+\Delta_{c}^{2}}$.

The gain-absorption coefficient on transition $|2\rangle \leftrightarrow$ $|1\rangle$ is proportional to the imaginary part of $\rho_{12}$, and will be positive if $\operatorname{Im}\left(\rho_{12}\right)>0$.

Without the incoherent pump $(\Lambda=0)$, it is easy to get $\rho_{22}=\rho_{33}=\rho_{44}=0$, and $\rho_{11} \cong 1$ from Eq. (3), thus Eq. (4) can be simplified as:

$$
\rho_{12}=-\frac{i \Omega_{p}}{\gamma_{21}+i \Delta_{p}+\frac{\left|\Omega_{c}\right|^{2}}{\gamma_{41}+i\left(\Delta_{p}+\Delta_{c}\right)}}
$$

From Eq. (6), we can see that the numerator of $\rho_{12}$ could never be zero, and the term $\left|\Omega_{c}\right|^{2} /\left(\gamma_{41}+i \Delta_{p}\right)$ in the denominator leads to resonant absorption reduction, which is commonly known as the electromagnetically induced transparency (EIT) phenomenon.

Without the coherent field $\left(\Omega_{c}=0\right)$, it is found from Eq. (4) that the negative contribution of the second term tends to zero and the EIT phenomenon disappears, so this system degenerates into a pure three level light amplification system. With the restriction of $\Omega_{c}=0$, from Eqs. (3) and (4), we can get:

$$
\begin{aligned}
& \rho_{22}=\frac{\Lambda \Gamma_{32} /\left(\Lambda+\Gamma_{31}+\Gamma_{32}\right)}{\Gamma_{21}} \rho_{11} \\
& \rho_{33}=\Gamma_{21} \frac{\rho_{22}}{\Gamma_{32}} \\
& \rho_{44}=0 \\
& \rho_{12}=\frac{i \Omega_{p}\left(\rho_{22}-\rho_{11}\right)}{\gamma_{12}+i \Delta_{p}}
\end{aligned}
$$

From Eq. (7), it is obvious that the probe can be amplified when the population inversion between level $|2\rangle$ and level $|1\rangle$ is established at the incoherent pumping rate $\Lambda>\Gamma_{21}\left(\Gamma_{31}+\Gamma_{32}\right) /\left(\Gamma_{32}-\Gamma_{21}\right)$.

When both the incoherent pumping and the coherent field are in action, the probe gain is contributed by the incoherent pump $\left(\propto\left(\rho_{22}-\rho_{11}\right)\right)$ and the atomic coherence $\left(\propto-\left|\Omega_{c}\right|^{2}\left(\rho_{22}-\rho_{44}\right)\right)$ (see Eq. (4)). With the incoherent pump process, level $|2\rangle$ could trap a large fraction of the population decayed nonradiatively from level $|3\rangle$, thus we can get rather high population inversion on transition $|2\rangle \leftrightarrow|1\rangle$. When the coherent field is included, it not only populates level $|4\rangle$ from level $|2\rangle$, but also splits level $|2\rangle$ into two dressed sublevels. If the positive contribution from the incoherent pump is larger than the negative contribution from the coherent field, the probe still can be amplified in the steady-state condition. However, if the negative contribution from the atomic coherence is dominant, the probe gain becomes smaller, and even changes into absorption. When the contribution from population difference equals that of the atomic coherence, the high index of refraction with zero absorption can be achieved in the steady-state condition.

\section{NUMERICAL RESULTS}

In the following, by numerical solutions, we discuss the mutual effects of light amplification and EIT to obtain a few important conclusions about the change of the gain profile. At the same time, we calculate how to combine the incoherent pumping and the coherent field to realize the high index of refraction with zero absorption.

Based on References [17,18], we can get the population spontaneous emission probabilities $\Gamma_{i j}$ of the $\mathrm{Er}^{3+}$ ions in $\mathrm{Er}^{3+}: \mathrm{YAG}$ crystals containing 0.52 at.\% concentrations of $\mathrm{Er}^{3+}$ ion at room temperature. So it is reasonable that we always chose the parameters as: $\Gamma_{31}=0.80 \Gamma_{21}, \Gamma_{32}=10.0 \Gamma_{21}, \Gamma_{41}=0.86 \Gamma_{21}$, $\Gamma_{42}=0.29 \Gamma_{21}, \Gamma_{43}=0.04 \Gamma_{21}$ in the following. In Reference [19], we have found the dephasing time of $\mathrm{Er}^{3-}$ :YAG crystal with an $\mathrm{Er}^{3+}$ concentration of $0.1 \%$ $\mathrm{T}_{2}=75 \mu \mathrm{s}$ on the ${ }^{4} \mathrm{I}_{15 / 2} \leftrightarrow{ }^{4} \mathrm{I}_{13 / 2}$ transition of $\mathrm{Er}^{3+}$ at $1526.97 \mathrm{~nm}$, the homogeneous line width $\Gamma_{h}=4.246$ $\mathrm{kHz}$. So it is reasonable for us to estimate the dephasing decay rate as $\gamma_{21}^{d p h}=\gamma_{31}^{d p h}=\gamma_{32}^{d p h}=\gamma_{41}^{d p h}=\gamma_{42}^{d p h}=$ $\gamma_{43}^{d p h}=15 \Gamma_{21}$. In this paper, all the parameters have been scaled by $\Gamma_{21}=239.1 \mathrm{~s}^{-1}$.

To illustrate the effects of atomic coherence on light amplification, we depict the variation of the gain of the probe field vs. the detuning of the probe in Fig. 2 when the incoherent pumping and the coherent field are in action. When $\Lambda=10.0$ and $\Omega_{c}=0.0$, it is obvious that there is only one gain peak at the position of $\Delta_{p}=0.0$, as shown by curve a. While when a resonant coherent field is exerted between $|2\rangle$ states and $|4\rangle$, state $|2\rangle$ will be split into two dressed sublevels, thus there are symmetric gain peaks at $\Delta_{p}= \pm \Omega_{c}$ 


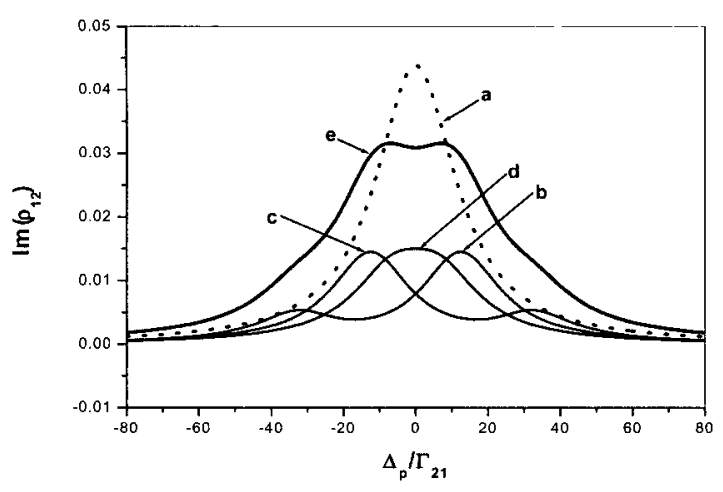

FIG. 2. Plots of the dimensionless probe gain coefficient $\operatorname{Im}\left(\rho_{12}\right)$ versus probe detuning $\Delta_{p} / \Gamma_{21}$ at different incoherent pumping rate and the coherent field. The curves $a, b$, c, d correspond to (1) $\Lambda=10.0, \Omega_{c}=0.0, \Delta_{c}=0.0$. (2) $\Lambda=10.0, \Omega_{c}=20.0, \Delta_{c}=20.0$. (3) $\Lambda=10.0, \Omega_{c}=20.0$, $\Delta_{c}=-20.0$. (4) $\Lambda=10.0, \Omega_{c}=6.0, \Delta_{c}=0.0$. The curve $\mathrm{e}$ is the combination of the curves $\mathrm{b}, \mathrm{c}$ and $\mathrm{d}$.

$[3,20]$. So we see that the probe gain, near the region $\Delta_{p}=0.0$, is dramatically changed. If the coherent field is not too strong, for example, $\Omega_{c}=6.0$, $\Delta_{c}=0.0$, we still observe only one probe gain peak, but the gain peak become low and flat, as shown by curve $d$ in Fig. 2. If we continue to increase the Rabi frequency of the coherent field, and change the detuning of the coherent field, for example, $\Omega_{c}=20.0$, $\Delta_{c}=20.0\left(\Delta_{c}=-20.0\right)$, we see that two gain peaks will separate and become asymmetric, as shown by curve b (c).

In the following a scheme is proposed to realize the equalization of the probe gain in the $\mathrm{Er}^{3+}$ Doped YAG crystal. As shown in Fig. 3, the probe beam passes though three identical Optical Couplers (OC) and the $\mathrm{Er}^{3+}: \mathrm{YAG}$ crystal successively. Each $\mathrm{Er}^{3+}:$ YAG crystal is pumped by a coherent field and an incoherent process with proper Rabi frequency and proper detuning. In Fig. 2, curves b, $\mathrm{c}$ and $\mathrm{d}$ correspond to the cases where the probe field passes through the first, the second and the third $\mathrm{Er}^{3+}$ :YAG crystal, respectively. Curve e corresponds to the case where the probe beam passes though the three same $\mathrm{Er}^{3+}: \mathrm{YAG}$ crystals successively. It is found that the gain pro-



FIG. 3. A scheme consisting of three $\mathrm{Er}^{3+}$ :YAG crystal with different coherent fields and incoherent pumpings where the probe passes through successively to realize the gain equalization of the probe beam.

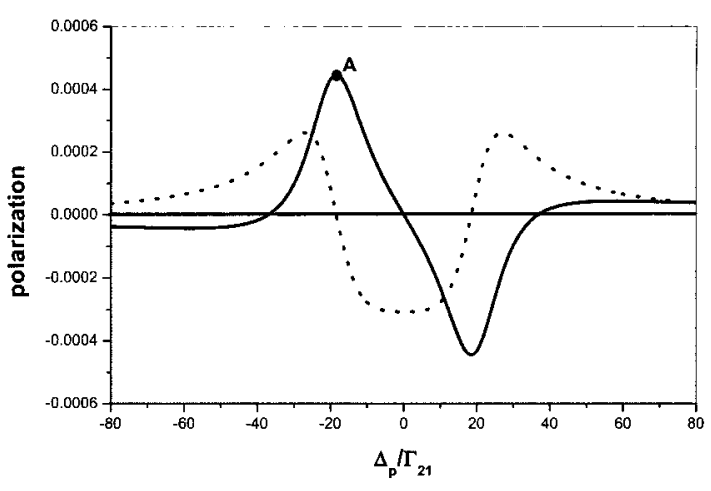

FIG. 4. Plots of the dimensionless Absorptive $\operatorname{Im} P$ (dotted) and Dispersive $\operatorname{Re} P$ (solid) parts of polarization versus probe detuning $\Delta_{p} / \Gamma_{21}$ at incoherent pumping rate $\Lambda=0.0$ and the Rabi frequency of the resonant coherent field $\Omega_{c}=85.0$. At $\Delta_{p}=0.0$, both $\operatorname{Im} P$ and $\operatorname{Re} P$ are equal to zero. The other parameters used are $\Gamma_{21}=239.1 \mathrm{~s}^{-1}$, $\Gamma_{31}=0.80 \Gamma_{21}, \quad \Gamma_{32}=10.0 \Gamma_{21}, \quad \Gamma_{41}=0.86 \Gamma_{21}$, $\Gamma_{42}=0.29 \Gamma_{21}, \Gamma_{43}=0.04 \Gamma_{21}, \Omega_{p}=1.0 \Gamma_{21}$.

file shown by curve e is much flatter and broader than that shown by curve a, and the height and the width of the equalized probe gain can be controlled by changing the coherent fields.

To observe how atomic coherence and interference effects lead to complete absorption cancellation and an ultrahigh index of refraction, we depict $\operatorname{Im} P$ and $\operatorname{Re} P$ of the probe field versus the detuning of the probe in Figs. 4 and 5 whether the incoherent pumping and the coherent field are in action or not, where the polarization is in the unit of $2 N \mu_{21}$ and the detuning of the probe field is in the unit of $\Gamma_{21}=239.1 \mathrm{~s}^{-1}$. When a resonant coherent field $\Omega_{c}=85.0$ is exerted between states $|2\rangle$ and $|4\rangle$ only, it was found that both $\operatorname{Im} P$ and $\operatorname{Re} P$ vanish [7] at the same detuning, i.e. at the point $\Delta_{p}=0.0$ in Fig. 4 . The main difference between the present model and that of Reference [7] is that only one incoherent pumping is used. The incoherent pum-

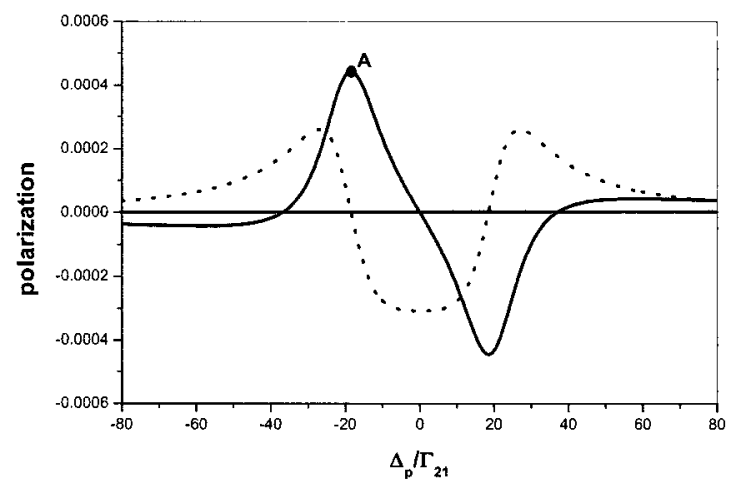

FIG. 5. The same as Fig. 2. $\Lambda=2.50, \Omega_{c}=20.0$, $\Delta_{c}=0.0$. At point A have zero $\operatorname{Im} P$ and nonzero $\operatorname{Re} P$. The other parameters used are same as those in Fig. 2. 


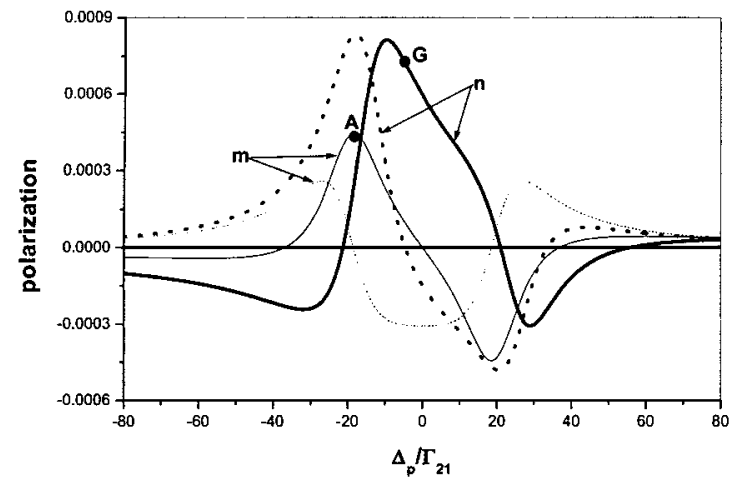

FIG. 6. The same as Fig. 2. The curve $m$ and $n$ correspond to (1) $\Lambda=2.50, \Omega_{c}=20.0, \Delta_{c}=0.0$. (2) $\Lambda=2.50$, $\Omega_{c}=20.0, \Delta_{c}=-10.0$, respectively. The other parameters used are same as those in Fig. 2.

ping and the coherent driving field are two crucial ingredients necessary for having zero $\operatorname{Im} P$ and large $\operatorname{Re} P$. If there is no incoherent pumping, both $\operatorname{Im} P$ and $\operatorname{Re} P$ vanish at $\Delta_{p}=0.0$ as shown in Fig. 4, in agreement with the results of References [7,11]. When the incoherent pumping rate $\Lambda=2.50$, and the Rabi frequency of the resonant coherent field $\Omega_{c}=20.0$, as shown in Fig. 5, it immediately becomes obvious that there is a test-field frequency that experiences both vanishing absorption and a high index of refraction at point A.

Next, we consider the case where the coherent field is not at resonance with the transition $|2\rangle \leftrightarrow|4\rangle$. It is found that one of the two symmetric gain peaks becomes higher than before, while the other one changes from positive into negative. Simultaneously, a higher index of refraction with vanishing absorption is obtained. In Fig. 6, with $\Lambda=2.50$ and $\Omega_{c}=20.0$, we find that, when the detuning of the coherent field $\Delta_{c}$ is changed from 0 to -10.0 , the index of refraction with zero absorption at point $\mathrm{G}$ in curve $\mathrm{n}$ becomes much higher than that at point $\mathrm{A}$ in curve $\mathrm{m}$. So the high index of refraction with zero absorption can be modified by the incoherent pumping rate $\Lambda$, the detuning $\Delta_{c}$ and the Rabi frequency $\Omega_{c}$ of the coherent field.

\section{CONCLUSIONS}

In this paper, a four-level model is constructed in which the gain of the traditional light amplification system and the high index of refraction with zero absorption can be modified by the quantum interference caused by the coherent field. Effects of quantum interference on the probe field gain are presented. At the same time, the origin of the high index of refraction is also analyzed. It is found that the maximum value of the index of refraction with zero absorption can be modified by the incoherent pump and the coherent field. In particular, it should be pointed out that a higher index of refraction with zero absorption is easily achieved when the coherent field is off resonance.

*Corresponding author : hfzhang@nju.edu.cn.

\section{REFERENCES}

[1] A. Kasapi, M. Jain, G. Y. Yin, and S. E. Harris, "Electromagnetically induced transparency: Propagation dynamics," Phys. Rev. Lett., vol. 74, no. 13, pp. 24472450, 1995.

[2] M. Xiao, Y.-Q. Li, S.-Z. Jin, and J. G.-B., "Measurement of dispersive properties of electromagnetically induced transparency in rubidium atoms," Phys. Rev. Lett., vol. 74, no. 5, pp. 666-669, 1995.

[3] K. J. Boller, A. Imamoğlu, and S. E. Harris, "Observation of electromagnetically induced transparency," Phys. Rev. Lett., vol. 66, pp. 2593-2596, 1991.

[4] J. G.-B., Y.-Q. Li, S.-Z. Jin, and M. Xiao, "Electromagnetically induced transparency in ladder-type inhomogeneously broadened media: Theory and experiment," Phys. Rev. A, vol. 51, no. 1, pp. 576-584 1995.

[5] S. E. Harris, "Lasers without inversion: Interference of lifetime-broadened resonances," Phys. Rev. Lett., vol. 62, pp. 1033, 1989; M. O. Scully, S.-Y. Zhu, and A. Gavrielides, "Degenerate quantum-beat laser: Lasing without inversion and inversion without lasing," Phys, Rev. Lett., vol. 62, no. 24, pp. 2813-2816, 1989.

[6] J. Gao, C. guo, X. Guo, G. Jin, P. Wang, J. Zhao, H. Zhang, J. Yun, D. Wang, and D. Jiang, "Observation of light amplification without population inversion in sodium," Opt. Commun., vol. 93, pp. 323-327, 1992; J.-Y. Gao, H.-Z. Zhang, H.-F. Cui, X.-Z. Guo, Y. Jiang, P.-W. Wang, G.-X. Jin, and J.-S. Li, "Inversionless light amplification in sodium," Opt. Commun., vol. 110, pp. 590-594, 1994.

[7] M. O. Scully and S.-Y. Zhu, "Ultra-large index of refraction via quantum interference," Opt. Commun., vol. 87, pp. 134-138, 1992.

[8] M. Fleischhauer, C. H. Keitel, and M. O. Scully, "Resonantly enhanced refractive index without absorption via atomic coherence," Phys. Rev. A, vol. 46, no. 3, pp. 1468-1487, 1992.

[9] A. S. Zibrov, M. D. Lukin, L. Hollberg, D. E. Nikonov, M. O. Scully, H. G. Robinson, and V. L. Velichansky, "Experimental demonstration of enhanced index of refraction via quantum coherence in Rb," Phys. Rev. Lett., vol. 76, no. 21, pp. 3935-3938, 1996.

[10] Y. Zhu, M. Xiao, and Y. Zhao, "Intensity characteristics of inversionless lasers from induced atomic coherence," Phys. Rev. A, vol. 49, no. 5, pp. 4016-4023, 1994.

[11] S. E. Harris, J. E. Field, and A. Imamoğlu, "Nonlinear optical processes using electromagnetically induced transparency," Phys. Rev. Lett., vol. 64, no. 10, pp. 1107-1110, 1990.

[12] D. Wang, J. Y. Gao, J. H Xu, G. C. La Rocca, and F. Bassani, "Electromagnetically induced two-photon 
transparency in rubidium atoms," Europhys. Lett., vol. 54 , no. 4 , pp. 456,2001

[13] Y. Zhao, C. Wu, B.-S. Ham, M. K. Kim, and E. Awad, "Microwave induced transparency in ruby," Phys. Rev. Lett., vol. 79, no. 4, pp. 641-644, 1997.

[14] B. S. Ham, P. R. Hemmer, and M. S. Shahriar, "Efficient electromagnetically induced transparency in a rare-earth doped crystal," Opt. Commun., vol. 144, p. $227,1997$.

[15] H. Xu, Z. Dai, and Z. Jiang, "Effect of concentration of the $\mathrm{Er}^{3+}$ ion on electromagnetically induced transparency in $\mathrm{Er}^{3+}$ :YAG crystal," Phys. Lett. A, vol. 294, pp. 19-25, 2002.

[16] G. B. Serapiglia, E. Paspalakis, C. Sirtori, K. L. Vodopyanov, and C. C. Phillips, "Laser-induced quantum coherence in a semiconductor quantum well," Phys. Rev. Lett., vol. 84, no. 5, pp. 1019-1022, 2000.
[17] Q. Y. Wang, S. Y. Zhang, and Y. Q. Jia, "Effect of the concentration of the $\mathrm{Er}^{3+}$ ion on the spectral intensity parameters of Er:YAG crystals," Journal of Alloys and Compounds, vol. 202, pp. 1-5, 1993.

[18] V. I. Zhekov, T. M. Murina, A. M. Prokhorov, M. I. Studenikin, S. Georgescu, V. Lupei, and I. Ursu, "Cooperative process in $\mathrm{Y}_{3} \mathrm{Al}_{5} \mathrm{O}_{12}: \mathrm{Er}^{3+}$ crystals," Sov. J. Quantum Electron, vol. 16, no. 2, pp. 274-276, 1986.

[19] Y. Sun, C. W. Thiel, R. L. Cone, R. W. Equall, and R. L. Hutcheson, "Recent progress in developing new rare earth materials for hole burning and coherent transient applications," J. Lumin., vol. 98, pp. 281$287,2002$.

[20] J. E. Field, K. H. Hahn, and S. E. Harris, "Observation of electromagnetically induced transparency in collisionally broadened lead vapor," Phys. Rev. Lett., vol. 67 , no. 22 , pp. 3062-3065, 1991. 